\title{
ANATOMY OF THE VEIN OF LABBE: A CADAVERIC STUDY
}

\section{Ashwini C Appaji *1, M urali Mohan 2, Roopa Kulkarni ${ }^{3}$, R N Kulkarni 4.}

${ }^{* 1}$ Associate professor of Anatomy, M S Ramaiah Medical College, Bangalore, Karnataka, India.

${ }^{2}$ Consultant Neurosurgeon, Sparsh Hospitals, Bangalore, Karnataka, India.

${ }^{3}$ Principal, Professor of Anatomy, K V G M edical College, Sulia, Dakshina Kannada, Karnataka, India.

${ }^{4}$ Professor of Anatomy (Retired), M S Ramaiah M edical College, Bangalore, Karnataka, India.

\section{ABSTRACT}

Background: Vein of Labbe is the major inferior anastomotic channel that connects the middle cerebral vein to the transverse sinus. It drains most of the temporal lobe and is clinically very important as injury to this vein would result in venous infarct of the temporal and parts of parietal lobe leading to not only functional deficits such as aphasia, memory and cognitive dysfunction but also can result in life threatening brain swelling.

Materials and Methods: 64 anatomical formalin embalmed cadaveric specimens of the head were used for the study. The pattern of formation, the number, course, dominance, relationships and the pattern of termination were noted.

Results: The vein of Labbe had a variable course on the lateral surface of the temporal lobe. It drained into the transverse sinus commonly in the area of pre-occipital notch. The vein of Trolard was prominent in $70.5 \%$ of specimen and the vein of Labbe was prominent in $14.7 \%$ and both were equidominant in $14.7 \%$. The variation in vein of Labbe noted was a dural course before draining into the transverse sinus.

Conclusion: The vein of Labbe is a constant vein present on the supero-lateral surface of the cerebral hemispheres. The variability of the course of vein through the dura and its drainage into transverse sinus at variable sites and other nearby sinuses indicates its vulnerability to injury during various craniotomy procedures, hence the anatomical knowledge of the vein of Labbe is of utmost importance for the Neurosurgeon to plan safe surgical approaches.

KEY WORDS: Vein Of Labbe, Inferior Anastomotic Vein, Temporal Lobectomy.

Address for Correspondence: Dr. Ashwini C Appaji, Associate Professor of Anatomy, M SRamaiah Medical College, Bangalore 560054, Karnataka, India. E-M ail: ashwinishivaprasad@ gmail.com

\section{Access this Article online}

\section{Quick Response code}

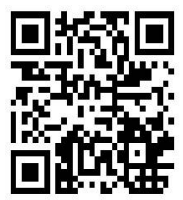

DOI: $10.16965 /$ ijar.2016.505

Web site: International Journal of Anatomy and Research

ISSN 2321-4287

www.ijmhr.org/ijar.htm

Received: 09 Dec 2016

Peer Review: 13 Dec 2016

Revised: None
Accepted: 24 Dec 2016

Published (0): 31 Jan 2017

Published (P): 31 Jan 2017

\section{INTRODUCTION}

The venous drainage of the cerebrum occurs by two sets of veins which consist of superficial (external) and deep (internal) veins. There are fine veins in the substance of the brain, which form a plexus in the pia mater and drain into the larger veins, called the cerebral veins. The cerebral veins drain in to the dural venous sinuses after passing through the subarachnoid space [1-8].

The superficial veins are divided in to superior, superficial middle and inferior groups of cerebral veins. The superior cerebral veins are about 10 to 15 in number. The superficial middle 
cerebral vein is usually single and drains anteriorly in to the cavernous sinus. Its posterior end divides in to superior anastomotic vein (Vein of Trolard or great anastomotic vein) and inferior anastomotic vein (vein of Labbe or small anastomotic vein).

Vein of Labbe is the major inferior anastomotic channel that connects the middle cerebral veins to the transverse sinus. It drains most of the temporal lobe and is clinically a very important vein as thrombosis or avulsion of the vein would result in venous infarct of the temporal and parts of parietal lobe leading to not only functional deficits such as aphasia, memory and cognitive dysfunction but also can result in life threatening brain swelling resulting in tentorial herniation due to the proximity of the mesial temporal structures to the tentorial hiatus [7].

The accurate knowledge of the presence, origin, course, numbers and dominance of vein of Labbe is vital during cranial surgeries involving the middle cranial fossa approaches, as this vein bridges between the inferior surface of temporal lobe of cerebrum and transverse dural venous sinus. Any attempt at retraction of the temporal lobe without the knowledge of the vein of Labbe can result in inadvertent injury to the vein. This vein is usually injured or lacerated during the incision of the dura mater. The vein may undergo thrombosis when it is excessively and forcibly retracted [4].

\section{MATERIALS AND METHODS}

34 anatomical specimens of the head (right and left side - 64 sides) were used for the study over four years (2010-2014). The cadaver heads were all formalin embalmed. The sources of specimen were from the department of Anatomy of M SRamaiah M edical College and International Medical School, Malaysian Science University, M.S.Ramaiah Campus, Bangalore.

The line of removal of the calvaria went through the supra orbital margin, upper margin of the tragus and $2 \mathrm{cms}$ below the external occipital protuberance (Fig.1). Once the markings were made, calvaria was removed by careful chisel and hammering along the incision without injuring the dura mater. The dura mater was incised in a criss-cross manner along the sagittal and coronal plane. The dura mater was then carefully reflected to visualise the lateral surface of the cerebrum, the superior and inferior anastomotic veins [5]. Then measurements of the length of the vein of Labbe from its formation/beginning to its termination were taken (cisternal segment). The pattern of formation and the number of tributaries were noted. At its termination, the pattern of drainage channels and the dural venous sinus of drainage were studied. The sub dural course length of the vein of Labbe was measured from the point of the vein leaving the brain to the point of draining into the venous sinus. Both the vein of Trolard and the vein of Labbe were observed for dominance. The above mentioned measurements and observations were made on either sides of the brain. Every measurement was taken thrice by the same observer to avoid intra observer variation and average of the three measurements was taken as results.

Fig.1: Marking for removal of calvaria.

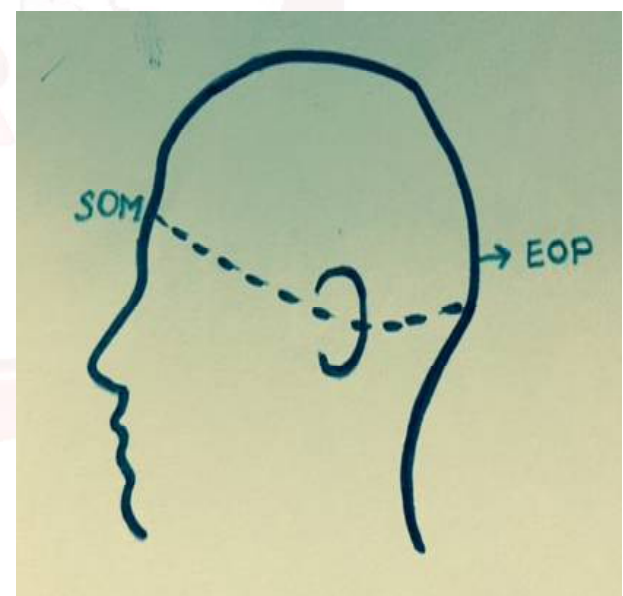

SOM - Supraorbital margin, EOP- External Occipital Protuberance

The following table gives the observations made.

\begin{tabular}{|c|c|}
\hline Sl. No. & Measurement/ Observation \\
\hline 1 & Point of origin from the Pterion \\
\hline 2 & Point of exit from the pterion. \\
\hline 3 & Cisternal segment \\
\hline 4 & Subdural segment \\
\hline 5 & $\begin{array}{c}\text { Point/Area where the vein drained into the } \\
\text { sinus }\end{array}$ \\
\hline 6 & No. of veins draining in this area \\
\hline 7 & Dural venous sinus into which it drained \\
\hline 8 & $\begin{array}{c}\text { Prominence of vein in relation to vein of } \\
\text { Trolard }\end{array}$ \\
\hline 9 & Variations if any \\
\hline
\end{tabular}


Photos were taken for each measurement using Sony $16 \mathrm{M} \mathrm{P}$ camera. The fevicryl paint and brush was used to mark the anatomical landmarks for the incisions and during taking of measurements.

\section{RESULTS}

Table 1: Showing the measurements and observations related to vein of Labbe.

\begin{tabular}{|c|c|c|c|}
\hline SI. No. & Measurement/Observation & Right side & Left side \\
\hline 1 & $\begin{array}{l}\text { Point of origin from the Pterion } \\
\text { (average) }\end{array}$ & $2.81 \mathrm{cms}$ & $2.99 \mathrm{cms}$ \\
\hline 2 & Point of exit from the pterion. & $8.22 \mathrm{cms}$ & $7.46 \mathrm{cms}$ \\
\hline 3 & Cisternal segment & $7.24 \mathrm{cms}$ & $6.60 \mathrm{cms}$ \\
\hline 4 & Subdural segment & $1.04 \mathrm{cms}$ & $1.02 \mathrm{cms}$ \\
\hline \multirow{5}{*}{5} & $\begin{array}{l}\text { Dural venous sinus into which it } \\
\text { drained }\end{array}$ & Right side & Left side \\
\hline & Transverse sinus & $28(82.35 \%)$ & $31(91.1 \%)$ \\
\hline & Superior petrosal sinus & $4(11.76 \%)$ & $1(2.9 \%)$ \\
\hline & $\begin{array}{l}\text { unction of transverse and superior } \\
\text { petrosal sinus }\end{array}$ & 0 & $2(5.88 \%)$ \\
\hline & Others ${ }^{*}$ & $2(5.88 \%)$ & 0 \\
\hline \multirow{9}{*}{6} & No. of veins draining in this area & Right side & Left side \\
\hline & 1 & $12(35.29 \%)$ & $13(38.23 \%)$ \\
\hline & 2 & $7(20.5 \%)$ & $7(20.5 \%)$ \\
\hline & 3 & $7(20.5 \%)$ & $3(8.88 \%)$ \\
\hline & 4 & $5(14.7 \%)$ & $8(23.52 \%)$ \\
\hline & 5 & $2(5.88 \%)$ & $2(5.88 \%)$ \\
\hline & 6 & 0 & 0 \\
\hline & 7 & 0 & $1(2.94 \%)$ \\
\hline & 8 & $1(2.94 \%)$ & 0 \\
\hline \multirow{4}{*}{7} & $\begin{array}{l}\text { Point/Area where the vein drained } \\
\text { into the sinus }\end{array}$ & Right side & Left side \\
\hline & Pre Occipital Notch (PON) & 26 & 22 \\
\hline & Anterior to PON & 6 & 8 \\
\hline & Posterior to PON & 2 & 4 \\
\hline \multirow{4}{*}{8} & $\begin{array}{l}\text { Prominence of vein in relation to } \\
\text { vein of Trolard }\end{array}$ & Right side & Left side \\
\hline & Vein of Trolard & 24 & 24 \\
\hline & Vein of Labbe & 5 & 5 \\
\hline & Equidominant & 5 & 5 \\
\hline \multirow{2}{*}{9} & Variations if any & Right side & Left side \\
\hline & Dural segment of vein of Labbe & $6(17.64 \%)$ & $3(8.82 \%)$ \\
\hline
\end{tabular}

Others: near confluence of sinus but into transverse sinus, junction of transverse sinus and sigmoid sinus. PON- Preoccipital notch.

Variations: The common variation noted was the dural course of the vein of Labbe before drainage.

Normally, the vein of Labbe left the cortical surface of the brain went through a subdural course and then pierced the dura to drain into the transverse sinus. But in the above mentioned dural segment variation, the vein left the cortical surface of the brain and entered the dura, traversed for a variable length within the dura and reached the desired dural venous sinus. Whatever was the course in the dura, all the dural segments drained into the transverse sinus. The area where it drained into the transverse sinus was the pre occipital notch more near the posterior end except in a few cases where it drained almost near the tip of the occipital lobe.

\section{DISCUSSION}

The vein of Labbe is widely described as the inferior anastomotic vein which connects the superficial middle cerebral vein to the transverse sinus. It is the largest venous channel which runs from the sylvian fissure to the transverse sinus (Fig. 2). However, in reality a wide range of variations from its formation, course, area of drainage and pattern of termination are possible. It is very essential for a surgeon to be aware of such variations as any inadvertent injury to this vein can result in poor surgical outcome including mortality.

Fig. 2: Showing the vein of Labbe in two specimen.

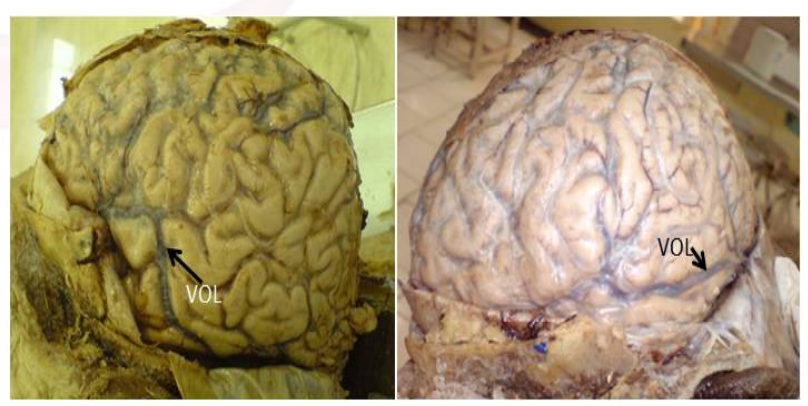

Fig. 3: Showing the drainage areas of vein of Labbe.

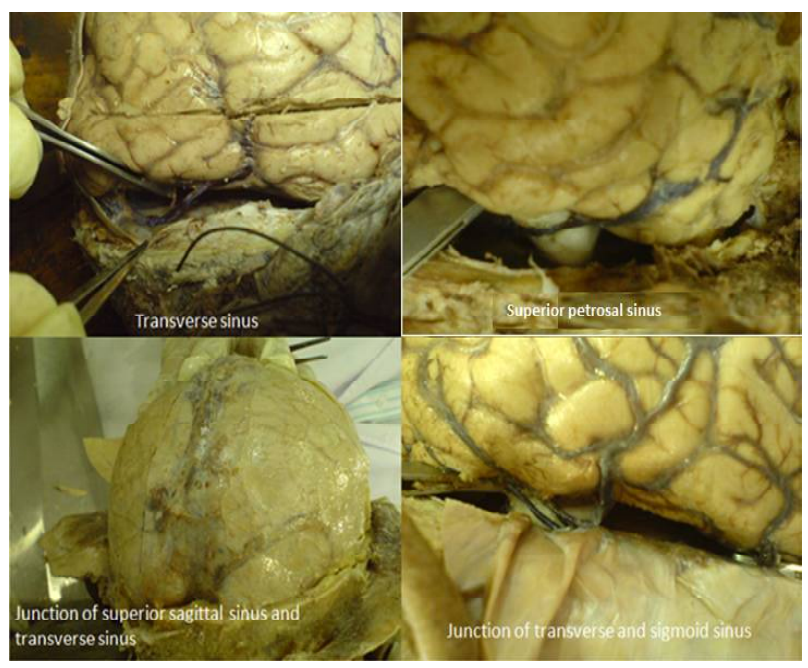


The vein of Labbe usually drained most of the temporal lobe on the lateral surface. It not only drains the area adjacent to the lateral sulcus but also drains the medial and inferior parts of temporal lobe in $80 \%$ of cases observed in the cadavers used for dissection. This knowledge is important while planning several surgical procedures such as temporal lobectomy in refractory temporal epilepsy especially when the vein is located anteriorly.

Vein of Labbe- its extent: The vein of Labbe extends from the end of posterior ramus of lateral sulcus to the transverse sinus, usually in the area of pre occipital notch or at the lateral edge of the petrous temporal bone where the transverse sinus continues as the sigmoid sinus. The point of origin (measured from the pterion) was very varied and ranged from $1.8 \mathrm{~cm}$ to $8.3 \mathrm{~cm}$ on the left and $1.9 \mathrm{~cm}$ to $6.6 \mathrm{~cm}$ on the right side. It was inferred that the point of origin of vein of Labbe was variable and lies on the posterior ramus of lateral sulcus.

When the point of exit was measured from the pterion, it was observed that the point of exit was more or less consistent and was located in the area of the pre-occipital notch. The mean distance of the point of exit to the pterion was $8.22 \pm 1.67 \mathrm{~cm}$ on the right and $7.46 \pm 1.66 \mathrm{~cm}$ in the left side. The point of exit was more consistent in location than the point of origin of the vein of Labbe. This could be because of the constant location of transverse sinus which is the drainage point for the vein of Labbe. The formation of the superficial middle cerebral vein was more inconsistent and dependent on the union of the vein of Labbe and the vein of Trolard.

The cisternal segment was measured along the course of the vein of Labbe in the subarachnoid space from the point of origin to the point of exit. The average cisternal segment measured was $6.78 \pm 1.15 \mathrm{cms}$ on the right and $6.25 \pm 1.57$ cms on the left side. This indicates that the cisternal segment was more or less similar on either side. The vein usually traversed along the lateral surface of the temporal lobe before exiting the subarachnoid space to become the subdural segment and later piercing the dura matter to drain into the transverse sinus. The vein terminated into the transverse sinus in the area of pre-occipital notch most often $(70.58 \%$ on the right and $64.70 \%$ on the left side). The other areas of point of exit have been tabulated in (Table No.1)

The subdural segment was the course of the vein of Labbe after exiting the brain surface till it reached the dura matter. The mean length of subdural segment was $1.01 \pm 0.35 \mathrm{~cm}$ on the right and $1.05 \pm 0.4 \mathrm{~cm}$ on the left side. Out of the 34 specimens, 11 on the right and 14 on the left had a very short segment measuring less than $5 \mathrm{~mm}$ and directly terminated in to the sinus. It can be concluded that the vein of Labbe had more variations at the point of its origin than at the point of drainage into the transverse sinus.

Sinus of drainage: The literature mentions the standard sinus into which the vein of Labbe drains is the transverse sinus. In the present study, most commonly the vein of Labbe drained into the transverse sinus except for a few. Other sites of termination included superior petrosal sinus, junction of superior petrosal sinus and transverse sinus (Fig. 3) (table no.1). The variations were more common on the right side in our study. It is very important to know the pattern of drainage of the vein of Labbe as it can be compromised during surgical procedures involving tentoriotomy and petrosal approaches. Fig. 4: Showing multiple veins draining in the area of termination of vein of Labbe into transverse sinus.

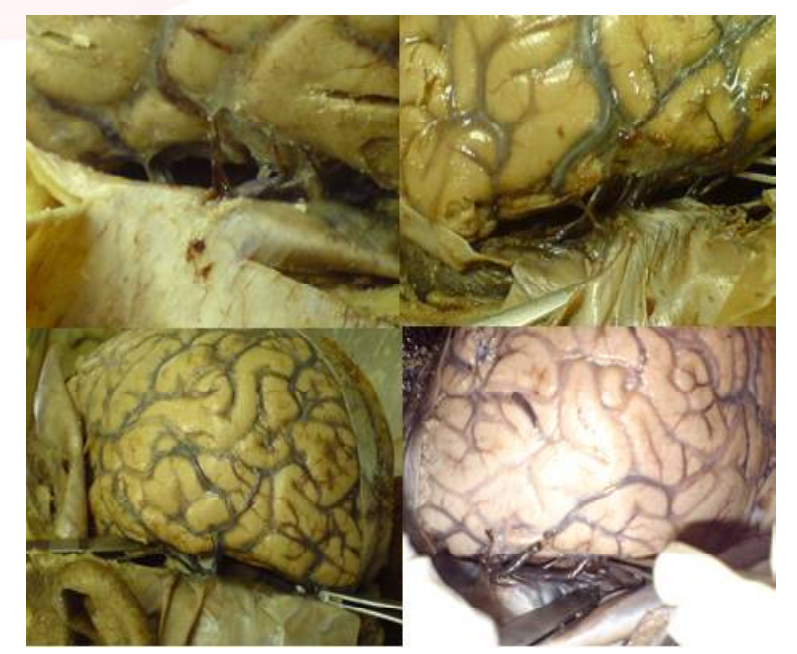

Number of veins draining in the vicinity of pre-occipital notch: It has been observed that the vein of Labbe drained in the area of pre-occipital notch. Few of them drained anterior and posterior to pre-occipital notch (Fig. 5). Along with vein of Labbe, there were other veins from the occipital lobe and inferior surface of the temporal lobe draining into the 
transverse sinus in this region (Table No.1, Fig. 4). The number of veins draining along with the vein of Labbe was ranging from 1 to 8 inclusive of the vein of Labbe. Only $35.29 \%$ to $38.23 \%$ of the cadaver specimen showed a single vein of Labbe draining into the transverse sinus in the vicinity of the pre-occipital notch.

Fig. 5: Showing the point of drainage of vein of LabbePON (C), anterior to PON (A) and posterior to PON (B).

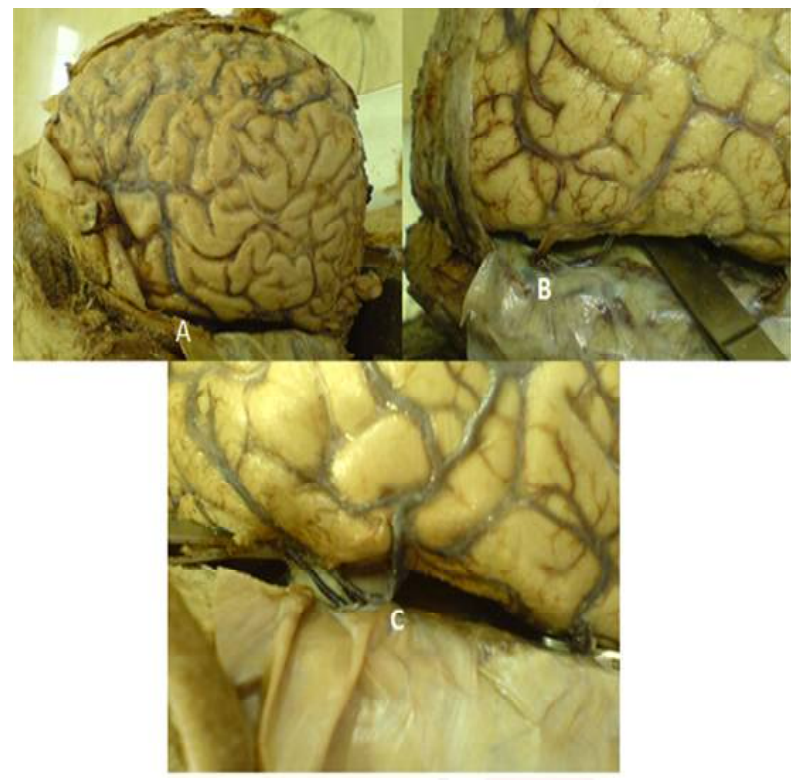

Prominence of Vein of Labbe: The superficial middle cerebral vein communicates with the superior sagittal sinus and the transverse sinus through the vein of Trolard and the vein of Labbe respectively. It has also been observed that the caliber of vein of Trolard was inversely proportional to the calibre of the vein of Labbe. Whenever the vein of Trolard was prominent, the vein of Labbe was thin in caliber and was not prominent.

In a radiographic study, it has been observed that the vein of Labbe was prominent in $40 \%$ of cases, the vein of Trolard in $32 \%$ and the superficial middle cerebral vein in $8 \%$ of cases. Both the vein of Labbe and Trolard was equally prominent in $11 \%$ of cases [2]. Another angiographic study involving 59 patients revealed the prominence of vein of Labbe in $31 \%$ of the cases and more so, on the left side [6]. In the present study, the vein of Trolard was prominent in $70.58 \%$ of specimen and the vein of Labbe in $14.70 \%$. The vein of Labbe was dominant more on the left side than the right side. In the remaining $14.72 \%$ of specimen, both the veins were of equal calibre (Fig. 6, Table No.1)
Fig. 6: Showing the prominent vein of Trolard and vein of Labbe.

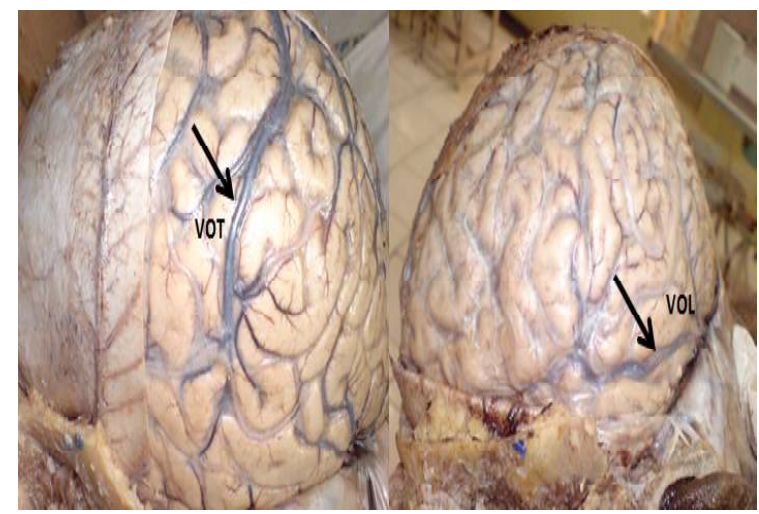

In cases where the vein of Labbe is predominant, utmost caution needs to be observed during surgery to prevent iatrogenic injury to the vein as it can lead to complications like hemiparesis, speech disturbance and at times life threatening brain swelling.

\section{Variations in the course of Vein of Labbe:}

The only variation observed in the course of the vein of Labbe was dural segment. Normally, the vein of Labbe traverses the lateral surface of the temporal lobe and communicates with the transverse sinus by piercing the dura immediately after leaving the subarachnoid space. In the present study, the vein of Labbe in around $17.64 \%$ on the right side and $11.76 \%$ on the left side traversed the dura over a variable length before draining into the venous sinuses (Table No.1). In about $9 \%$ of specimens on the right side, the vein of Labbe anastomosed with other veins before draining into the venous sinus. Based on the course of the dural segment, the vein of Labbe either drained into the transverse sinus near the lateral part of the petrous part of temporal bone (termination of transverse sinus) or into the transverse sinus near the confluence of sinuses (beginning of the transverse sinus) (Fig. 7).

In a study, the vein of Labbe drained into the transverse sinus via tentorial sinuses in $73 \%$ of cases and as a dural lacuna before draining to the transverse sinus in $23 \%$ of cases [3].

The knowledge of the dural course and the pattern of terminations are very important to plan surgical approaches as dural incision in this region can lead to venous injury resulting in intra and post-operative complications. 
Fig. 7: Showing the variant dural segment of vein of Labbe.

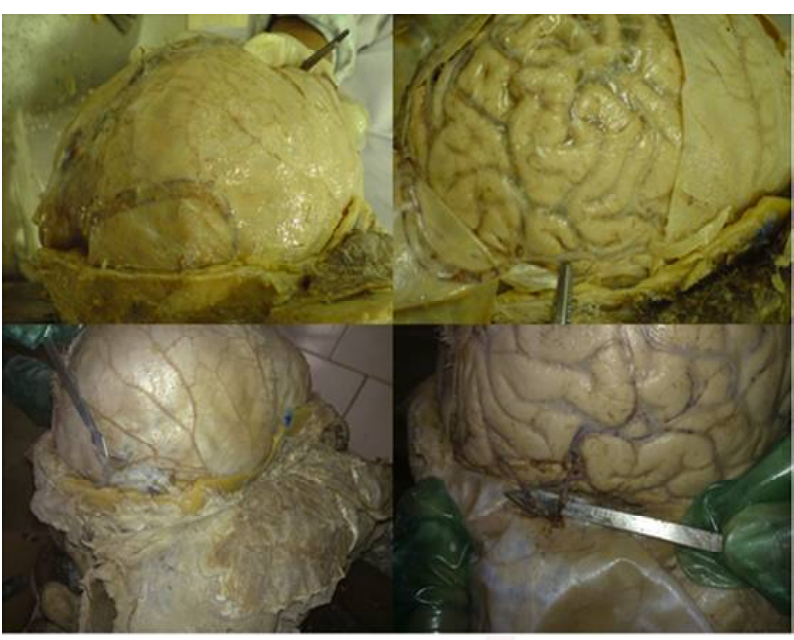

\section{CONCLUSION}

The vein of Labbe is a constant vein present on the supero-lateral surface of the cerebral hemispheres. Most often it drains the area around the lateral sulcus of the temporal lobe, but also drains the adjacent regions from parietal, inferior temporal and occipital lobe when it is dominant. The variability of the course of vein of Labbe through the dura and its drainage into transverse sinus at different variable sites and other nearby sinuses indicates its vulnerability to injury during various craniotomy procedures; hence the anatomical knowledge of the vein of Labbe is of utmost importance for the Neurosurgeon to plan safe surgical approaches.

\section{ABBREVIATIONS}

PON - Pre occipital notch

SOM - Supraorbital margin

EOP - External Occipital Protuberance

VOL - Vein of Labbe

\section{ACKNOW LEDGEM ENTS}

The authors thank the department of Anatomy, M S Ramaiah M edical College for their support and encouragement.

\section{Conflicts of Interests: None}

\section{REFERENCES}

[1]. Carpenter M B. Blood supply of Central Nervous System-Cerebral veins and venous siuses. Core Text of Neuroanatomy. $4^{\text {th }}$ Edition. Williams and Wilkins, Baltimore. 1991;455-461.

[2]. Di Chiro G. Angiographic patterns of cerebral convexity veins and superficial dural sinuses. AJR 1962;87:308-321.

[3]. Koperna T, Tschabitscher M , Knosp E. The termination of the vein of "Labbe" and its microsurgical significance. Acta Neu- rochir (Wien) 1992;118(34):172-17.

[4]. Lawrence R. Lustig and Robert K. Jackler, The vulnerability of Vein of Labbe during combined craniotomies of the middle and posterior fossae, Skull base Surgery. 1998;8(1): 1-9.

[5]. Romanes G J. Cunningham's manual of Practical anatomy. Volume -3 Head and Neck and Brain. $15^{\text {th }}$ edition. Oxford Medical Publications. Oxford. 2009;43-44.

[6]. Silva PS, Vilarinho A, Carvalho B, Vaz R. Anatomical variations of vein of Labbe- an angiographic study. Surg Radio Anat. 2014;36:769-773.

[7]. Sinnathamby C S. Central Nervous system. Last's Anatomy - Regional and Applied, $12^{\text {th }}$ edition, Elsevier Churchill Livingstone, 2011;473.

[8]. Standring S. Vascular Supply of Brain. Gray's Anatomy-The Anatomical basis of Clinical Practice. $41^{\text {st }}$ edition. Elsevier. Churchill Livingstone. 2016;280290.

How to cite this article:

Ashwini C Appaji, M urali M ohan, Roopa Kulkarni, R N Kulkarni. ANATOMY OF THE VEIN OF LABBE: A CADAVERIC STUDY. Int J Anat Res 2017;5(1):3451-3456. DOI: 10.16965/ijar.2016.505 